\title{
The Influence of Topical Prostaglandin Analogues in Inflammation After Selective Laser Trabeculoplasty Treatment
}

\author{
Marcelo Ayala and Enping Chen
}

\begin{abstract}
Purpose: Reducing intraocular pressure (IOP) seems to be the only treatment that slows progression in glaucoma. The IOP can be decreased by pharmaceutical treatment, laser [selective laser trabeculoplasty (SLT)] treatment, or surgery. Prostaglandin analogues have been postulated to share action mechanisms with SLT and to possibly diminish the effects of SLT treatment. The aim of the current study was to investigate the effects of prostaglandin analogues in inflammation and IOP reduction after SLT treatment.

Methods: Prospective nonrandomized study. One hundred and eighteen patients were included in the study. Inclusion criteria: Glaucoma (open-angle or pseudoexfoliation glaucoma) patients who will be treated with SLT. Inflammation was measured with a laser flare meter (Kowa FM-500). Measurements were made before SLT and then $2 \mathrm{~h}, 1$ week, and 1 month after SLT treatment. IOP was also checked at the same time intervals. The SLT treatment was performed over $90^{\circ}$. All patients were divided into two groups: those receiving prostaglandins analogues and those treated with nonprostaglandin analogues.

Results: Inflammation before and after SLT showed no significant difference between the groups at all the time intervals studied ( $t$-test, before: $P=0.16 ; 2 \mathrm{~h}: P=0.14$; 1 week: $P=0.12$; and 1 month: $P=0.36$ ). IOP reduction showed no significant difference between the groups ( $t$-test, $P=0.31$ ).

Conclusions: SLT treatment effects do not seem to be influenced by the use of prostaglandin analogues.
\end{abstract}

\section{Introduction}

G LAUCOMA Is a progressive neuropathy localized in the optic nerve that may lead to blindness. Reducing intraocular pressure (IOP) seems to be the only treatment that slows progression in glaucoma. There are several methods to reduce IOP: pharmaceutical treatment, laser treatment, and surgery. In the Glaucoma Laser Trial Follow-up study, after 7 years of follow-ups, patients who had undergone argon laser trabeculoplasty (ALT) had lower IOP than patients on pharmaceutical treatment. ${ }^{1,2}$ Laser treatment is classically performed when pharmaceutical treatment has failed. However, in selective laser trabeculoplasty (SLT), there is still not enough evidence to determine the effectiveness of laser trabeculoplasty compared to medication. ${ }^{3}$

The ALT was introduced about 30 years ago and involves the use of a blue/green argon laser (major wavelength peaks at $488 \mathrm{~nm}$ and $514 \mathrm{~nm}$ ) to treat the anterior part of the trabecular meshwork and thereby improves aqueous flow and thus reduces IOP. The SLT is a rather new treatment alter- native. It involves using a frequency-doubled, Q-switched Nd:YAG laser $(532 \mathrm{~nm})$ that delivers a low-energy, large spot, very brief pulse to selectively targeted cells of the trabecular meshwork. This laser application is thought to stimulate the pigmented trabecular meshwork cells and thus facilitates improved aqueous outflow. The SLT treatment may hold clinical advantages over the ALT treatment.

The ALT treatment induces inflammation in the anterior chamber due to the fact that the laser disrupts cells in the trabecular meshwork. Martinez-de-la-Casa et al., ${ }^{4}$ when using the laser flare meter (Kowa FM-500), reported lower inflammation in the anterior chamber when comparing SLT with ALT treatment. Little information is to be found in the literature regarding inflammation status in the anterior chamber after SLT treatment and the influence of medications on inflammation after SLT treatment.

Topical prostaglandin analogues have been demonstrated to be effective medications for lowering IOP. ${ }^{5-7}$ These agents have been shown to lower IOP mainly by increasing uveoscleral outflow. ${ }^{8-10}$ However, Alvarado et al. ${ }^{11}$ showed in a

Department of Glaucoma, St. Erik Eye Hospital, Karolinska Institutet, Stockholm, Sweden. 
translational model that prostaglandin analogues could have direct effects on the trabecular meshwork as well. Studies on the effects of medications and particularly prostaglandin analogue medications in SLT results are controversial. Scherer ${ }^{12}$ showed, in a retrospective study, a greater average IOP reduction after SLT when patients were treated with prostaglandin analogues. On the other hand, Alvarado et al. ${ }^{11}$ showed that prostaglandin analogues may interfere with SLT, thus decreasing SLT effects. Singh et al., ${ }^{13}$ in a retrospective study published in 2009, did not find that prostaglandin analogues affected outcomes after SLT.

The aim of the current study was to investigate the influence of prostaglandin analogues in inflammation and IOP reduction after SLT treatment.

\section{Methods}

\section{Study design and methods}

This was a prospective nonrandomized comparative study to assess the effects of prostaglandin analogues in inflammation and IOP reduction after SLT treatment.

Inclusion criteria: Patients suffering from glaucoma, primary open angle glaucoma (POAG), or pseudoexfoliation glaucoma (PXFG), who underwent SLT. If both eyes were treated, then one eye was chosen at random to be included in the study. Patients were included consecutively. All patients were treated with at least one kind of IOP-lowering topical medication.

Exclusion criteria:

- Patients treated with cortisone or immunosuppressive drugs.

- Patients suffering from ocular or systemic inflammatory diseases.

- Patients who cannot be treated with SLT due to the eye's characteristics (shallow anterior chamber) or bad collaboration.

- Patients treated with Pilocarpine (miosis makes laser flare measurements very difficult).

Patients coming to the Glaucoma Department at the St. Erik Eye Hospital for SLT treatment who fulfilled the inclusion criteria were asked to participate in the study. Patients were enrolled between January 2009 and December 2009. The study was approved by the Ethical Committee at the Karolinska Institutet (approval No. 2009/1). Informed consent was obtained. The authors confirm adherence to the tenets of the Declaration of Helsinki.

A comprehensive medical and ocular history was obtained. All patients were asked about present inflammatory diseases and treatments. Ophthalmological examination was performed before including patients in the study. Visual acuity, IOP measurements, optic nerve status, gonioscopy, and the presence or absence of exfoliation were registered. Visual acuity was recorded using a Snellen's chart. The IOP was measured using a Goldmann's applanation tonometer. Gonioscopy was performed in a darkroom using a goniolens with an undilated pupil, and the anterior chamber angle was classified into 0-IV (according to Shaffer) and pigmentation was classified into 0-3. Pupils were dilated and exfoliation was checked; exfoliation was registered as present or absent. Then, the optic nerve status was evaluated using a 90-D lens. IOP, slit-lamp, and laser flare measurements were made before SLT and then $2 \mathrm{~h}, 1$ week, and 1 month after SLT.
The SLT is a Q-switched, frequency-doubled Nd:YAG, wavelength around $532 \mathrm{~nm}$. The SLT uses a single pulse (pulse duration $3 \mathrm{~ns}$ ) and the spot's size is estimated to be $400 \mu \mathrm{m}$. In the current study, SLT treatment was performed over $90^{\circ}$ with the SLT Solo laser (Ellex) using some 25-30 spots applied to the trabecular meshwork. Traditionally, $180^{\circ}$ of the trabecular meshwork is treated, but a study published by Chen et al. ${ }^{14}$ showed similar effects when $90^{\circ}$ of the trabecular meshwork was treated. The initial energy used was $0.9 \mathrm{~mJ}$. The energy was increased or decreased until bubble formation appeared and was then decreased by $0.1 \mathrm{~mJ}$ for the remainder of the treatment. The energy used in the current study was in the range $0.9-1.1 \mathrm{~mJ}$.

All patients continue with the same medical treatment after SLT. No patient was treated with corticoids, NSAIDs, or apraclonidine before or after SLT treatment.

\section{Outcome parameters}

The primary outcome of this study was inflammation. Flare measurements were made with a laser flare meter (Kowa FM-500). The Kowa FM-500 is based on the measurement principle of detecting scattered laser light. The intensity of the scattered light (directly proportional to the amount of particle flare) is measured by a photo-multiplier, which generates an electric signal. Flare measurements in the study were made before SLT (baseline) and then $2 \mathrm{~h}$, 1 week, and 1 month following SLT.

The unit of measurement employed by the FM-500 is photon counts per millisecond ( $\mathrm{pc} / \mathrm{ms})$. A $1+$ seen in the slitlamp corresponds to a laser flare photometry value of 28.3 $\pm 4.8 \mathrm{pc} / \mathrm{ms}$ and a flare of $3+$ to a value of $82.5 \pm 5.2 \mathrm{pc} / \mathrm{ms}$ according to Guex-Crosier et al. ${ }^{15}$ Herbort et al. ${ }^{16}$ have shown that even normal eyes express a normal flare measured to be $4 \mathrm{pc} / \mathrm{ms}$. Laser flare measurements were made at least five times according to Shah et al. ${ }^{17}$ and the average value was calculated. The IOP was checked using a Goldmann's applanation tonometer (Haag Streit). Measurements were made at the same time intervals as for the flare measurements.

To analyze the effect of prostaglandin analogues on SLT efficacy, the eyes were categorized according to whether they received a topical prostaglandin analogue (travoprost, latanoprost, or bimatoprost) alone or in combination with other glaucoma medications in the patients' glaucoma pharmaceutical therapy before SLT treatment. The eyes that did not receive prostaglandin analogues included those treated with other topical agents (carbonic anhydrase inhibitors, $\alpha-2$ agonists, or $\beta$-blockers). All included patients were on pharmaceutical treatment for at least 3 months before SLT and continued with the same pharmaceutical treatment at least 1 month after SLT.

\section{Statistical considerations}

Laser flare: Measurements were made in both groups: those treated with prostaglandin analogues and those treated with other agents. A two-tailed $t$-test was performed to test the difference between the two groups at each time interval (before SLT and 2h, 1 week, and 1 month after SLT). Significance level was 5\%.

IOP: Measurements were compared before SLT (baseline) and 1 month after SLT. Measurements were made in both 
groups: those treated with prostaglandin analogues and those treated with other agents. A two-tailed $t$-test was performed to test the difference between the two groups. Significance level was $5 \%$.

Sample size was estimated, with alpha $=0.05$, power 0.95 , estimated standard deviation $(\mathrm{SD})=2$, and in order to detect a difference of $2 \mathrm{pc} / \mathrm{ms}$ between the groups, 26 patients were needed in each group. All statistical analysis was performed using STATA statistical software (Statacorp).

\section{Results}

\section{Demography}

A total of 118 eyes (patients) were identified during the study period, of which 78 eyes received prostaglandin analogues and 40 eyes received nonprostaglandin agents. All participants attended their appointments. The average age was 72.53 (SD: 10.3) years. The gender distribution was $46.25 \%$ men and $53.75 \%$ women. Diagnosis distribution: 46.25\% POAG and 53.75\% PXFG (see Table 1).

\section{Endpoints}

Inflammation: Flare was measured at the four different time intervals-before SLT and $2 \mathrm{~h}, 1$ week, and 1 month after SLT in eyes treated with prostaglandin analogues and eyes treated with nonprostaglandin analogues. No significant difference in flare was found between the two groups at any of the time intervals tested ( $t$-test, before: $P=0.16 ; 2 \mathrm{~h}$ : $P=0.14$; 1 week: $P=0.12$; and 1 month: $P=0.36$ ) (Fig. 1 ).

Intraocular pressure: The IOP reduction 1 month after SLT in the eyes treated with prostaglandin analogues was $6.35 \mathrm{mmHg}$, while the IOP reduction in the eyes treated with nonprostaglandin agents was $5.77 \mathrm{mmHg}$. The difference was not significant ( $t$-test, $P=0.31$ ) (Fig. 2).

\section{Discussion}

The current prospective study showed that prostaglandin analogues did not induce inflammation after SLT treatment when the eyes were treated over $90^{\circ}$. Information about inflammation after SLT treatment is scarce. One of the first studies published is the one written by Latina et al. ${ }^{18}$ with $180^{\circ}$ SLT showing that $83 \%$ of SLT-treated eyes exhibited mild-to-moderate inflammation, appearing within $1 \mathrm{~h}$ after treatment, decreasing by $24 \mathrm{~h}$ after treatment, and nearly resolved in all cases within 5 days. Martinez-de-la-Casa et al. $^{4}$ evaluated inflammation using the same device as the one used in this study, the Kowa laser flare meter, and found significantly lower flare readings after SLT than ALT.

The fact that the eyes were treated with $90^{\circ}$ SLT must be taken into consideration. In comparable studies, the eyes have been treated with $180^{\circ}$ SLT. The lower laser energy dose used in this study can in some way explain why there is no difference in inflammation between eyes treated with prostaglandin analogues and those not. However, all eyes (prostaglandin analogue users and nonusers) were treated with SLT in a similar manner using the same amount of laser energy.

Prostaglandins are common mediators in the inflammatory response. It has been postulated that prostaglandin analogues could induce inflammation in the eye. In vitro studies have shown that prostaglandins released by endothelial cells exhibit strong chemoattractant activity for neutrophils and macrophages ${ }^{19}$ and that latanoprost can significantly increase the expression of apoptotic markers. ${ }^{20}$

In vivo studies with cases of macular edema or uveitis have been described in patients at high risk for inflammatory reactions. ${ }^{21,22}$ No inflammation due to prostaglandin analogues was found in the current study when $90^{\circ}$ of the trabecular meshwork was treated with SLT. Inflammation pathways in the eye for prostaglandin analogues are not well described. The SLT treatment and prostaglandin analogues probably follow different inflammation pathways. Inflammation markers were not investigated in the current study. Further in vivo or in vitro studies are needed to elucidate the roll of different markers as well as inflammation pathways after SLT treatment.

With regard to the hypotensive effect of SLT, we found a significant IOP reduction after treatment. The absolute mean IOP reduction in all the treated eyes in the current study was $6.06 \mathrm{mmHg}$, from a baseline of 23.66 to $17.60 \mathrm{mmHg}$ after 1 month. These results correlate well with the findings published by Latina et al., ${ }^{18}$ who found a mean IOP reduction of about $6 \mathrm{mmHg} 4$ weeks (1 month) after SLT and then the mean IOP reduction decreased toward $5.8 \mathrm{mmHg}$ after 26 weeks. Several studies have been published after Latina's initial report. In general, these studies reported a mean IOP reduction in the range of $4-6 \mathrm{mmHg}$ in the short term (approximately 6 months). ${ }^{3}$ In the current study, no difference in IOP reduction was found when comparing prostaglandin analogue users with nonusers. The results are in line with previous results published by Singh et al. ${ }^{13}$ However, Scherer $^{12}$ described a difference in IOP reduction when

Table 1. Baseline Characteristics of the Subjects Included in the Study

\begin{tabular}{lccc}
\hline Characteristics & Prostaglandin $(\mathrm{n}=78)$ & Nonprostaglandin $(\mathrm{n}=40)$ & $\mathrm{P}$ value \\
\hline Age (years) (SD) & $73.47(8.17)$ & $71.60(12.44)$ & $18 / 22$ \\
Sex (male/female) & $38 / 40$ & $23.52(3.2)$ & $0.32^{\mathrm{a}}$ \\
IOP before SLT (mmHg) (SD) & $24.35(3.95)$ & $7.18(3.08)$ & $0.20^{\mathrm{b}}$ \\
Flare before SLT (pc/ms) (SD) & $8.98(4.8)$ & $26.9(4.8)$ & $0.48^{\mathrm{a}}$ \\
Average no. of spots per eye (SD) & $26.1(4.6)$ & $0.92(0.13)$ & $0.19^{\mathrm{a}}$ \\
Average dose per eye (mJ) (SD) & $0.86(0.11)$ & $18 / 22$ & $0.72^{\mathrm{a}}$ \\
Glaucoma diagnosis (POAG/PXF) & $38 / 40$ & $0.22^{\mathrm{a}}$ \\
\hline
\end{tabular}

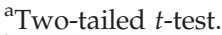

${ }^{\mathrm{b}}$ Fisher's exact test.

IOP, intraocular pressure; SLT, selective laser trabeculoplasty; POAG, primary open angle glaucoma; PXF, pseudoexfoliation; SD, standard deviation. 


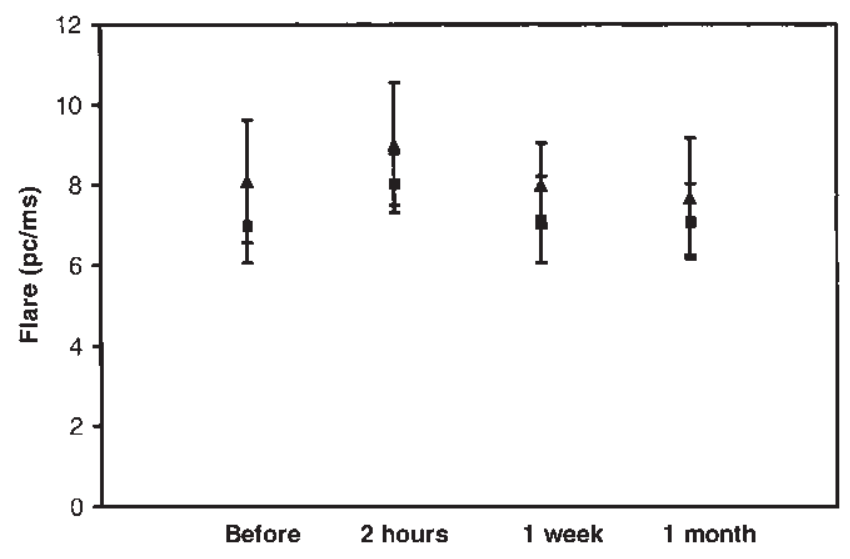

FIG. 1. Flare measurements in both groups at different time points after SLT treatment; the bars represent $95 \%$ confidence interval for the mean. $\boldsymbol{\Delta}$ represents the mean for prostaglandin analogues group. $\boldsymbol{\square}$ represents the mean for the nonprostaglandin analogues group ( $t$-test, before: $P=0.16$; 2 h: $P=0.14$; 1 week: $P=0.12 ; 1$ month: $P=0.36)$. SLT, selective laser trabeculoplasty.

comparing prostaglandin analogue users and nonusers when the baseline IOP was $17.75 \mathrm{mmHg}$. IOP levels before SLT treatment in different studies might be considered. The baseline IOP in the current study was $23.66 \mathrm{mmHg}$. The SLT efficacy decreases with lower IOP. ${ }^{23}$ It is possible to speculate that with a lower baseline IOP, the effects of eye drops are more prominent than the effects of SLT and this can explain the reason why Scherer found a difference between the groups. In an article published in 2009, Alvarado et al. ${ }^{11}$ described in a translational model (in vitro-in vivo) a possible common action mechanism for prostaglandin analogues and SLT. The authors concluded that prostaglandin analogues and SLT may share the same pathway (at the trabecular meshwork) for IOP reduction. The authors recommended that prostaglandin analogues might be discontinued before SLT treatment to achieve a good IOP reduction with SLT treatment. According to Alvarado et al., the IOP reduction after SLT treatment found in the prostaglandin analogue users group might be lower than in the group of nonusers. We did not find any difference. Comparisons among studies are difficult to perform due to the differences in the popu-

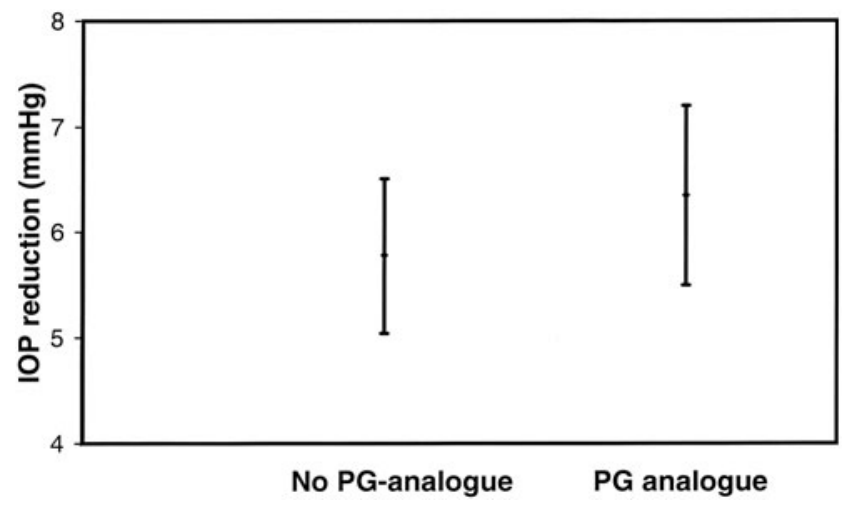

FIG. 2. Intraocular pressure (IOP) reduction comparing before SLT and 1 month after SLT treatment; the bars represent $95 \%$ confidence interval for the mean ( $t$-test, $P=0.31$ ). lations studied, diagnosis, follow-up periods, laser protocols, and so on.

One of the limitations of the study is the relatively short follow-up period of 1 month. The reason for this short follow-up is that the study focused principally on inflammation after SLT treatment. According to previous studies, ${ }^{4,18}$ inflammation decreases 1 month after SLT treatment. Further, after this time, some patients from each group have their glaucoma medication discontinued or SLT treatment repeated and this can alter the results. Considering IOP, several previous studies have indicated that IOP remains stable at 6 months ${ }^{18}$ and 18 months. ${ }^{24}$ Therefore, the 1 month IOP values are likely to be predictive of future IOP control.

Another possible limitation is selection bias. Patients were recruited at a tertiary care center where patients usually require more treatment. It is also necessary to consider the high number of patients exhibiting pseudoexfoliation. Pseudoexfoliation is a common finding among patients in Northern Europe. Pseudoexfoliation prevalence in northern Sweden is estimated to be $23 \%$ at 66 years and $61 \%$ at 87 years. ${ }^{25}$ In our study, $53.75 \%$ of all patients showed pseudoexfoliation, in part due to the high pseudoexfoliation prevalence in Sweden, but also due to the fact that pseudoexfoliative glaucoma generally needs additional therapy and SLT seems to be a good alternative. The high prevalence of pseudoexfoliation in our study population may introduce selection bias.

To the best of our knowledge, this is the first study investigating the influence of prostaglandin analogues and inflammation in the anterior chamber after SLT treatment treating just $90^{\circ}$, without any kind of antiinflammatory treatment before or after SLT, and using a quantitative method to measure inflammation in the anterior chamber. The overall lower inflammation levels found were probably due to the fact that a small amount of the trabecular meshwork was treated with consequently decreased total energy delivered.

\section{Acknowledgment}

Marcelo Ayala was supported by a grant from the Signhild Engkvist's Foundation.

\section{Author Disclosure Statement}

No competing financial interest exists.

\section{References}

1. The Glaucoma Laser Trial Research Group. The Glaucoma Laser Trial (GLT). Results of argon laser trabeculoplasty versus topical medicines. Ophthalmology 97:1403-1413, 1990.

2. The Glaucoma Laser Trial Research Group. The Glaucoma Laser Trial (GLT) and glaucoma laser trial follow-up study: 7. Results. Am. J. Ophthalmol. 120:718-731, 1995.

3. Realini, T. Selective laser trabeculoplasty: a review. J. Glaucoma 17:497-502, 2008.

4. Martinez-de-la-Casa, J., Garcia-Feijoo, J., Castillo, A., et al. Selective vs argon laser trabeculoplasty: hypotensive efficacy, anterior chamber inflammation, and postoperative pain. Eye 18:498-502, 2004.

5. Camras, CB. Comparison of latanoprost and timolol in patients with ocular hypertension and glaucoma: a six-month 
masked, multicenter trial in the United States. The United States Latanoprost Study Group. Ophthalmology 103:138-147, 1996.

6. Alm, A., and Stjernschantz, J. Effects on intraocular pressure and side effects of $0.005 \%$ latanoprost applied once daily, evening or morning. A comparison with timolol. Scandinavian Latanoprost Study Group. Ophthalmology 102:17431752, 1995.

7. Sihota, R., Saxena, R., Agarwal, H.C., and Gulati, V. Crossover comparison of timolol and latanoprost in chronic primary angle-closure glaucoma. Arch. Ophthalmol. 122:185189, 2004

8. Gabelt, B.T., and Kaufman, P.L. Prostaglandin F2 alpha increases uveoscleral outflow in the cynomolgus monkey. Exp. Eye Res. 49:389-402, 1989.

9. Bahler, C.K., Howell, K.G., Hann, C.R., Fautsch, M.P., and Johnson, D.H. Prostaglandins increase trabecular meshwork outflow facility in cultured human anterior segments. Am. J. Ophthalmol. 145:114-119, 2008.

10. Lim, K.S., Nau, C.B., O'Byrne, M.M., et al. Mechanism of action of bimatoprost, latanoprost, and travoprost in healthy subjects. A crossover study. Ophthalmology 115:790-795, E4, 2008.

11. Alvarado, J., Iguchi, R., Juster, R., et al. From the bedside to the bench and back again: predicting and improving the outcomes of SLT glaucoma therapy. Trans. Am. Ophthalmol. Soc. 107:167-181, 2009.

12. Scherer, W.J. Effect of topical prostaglandin analog use on outcome following selective laser trabeculoplasty. J. Ocul. Pharmacol. Ther. 23:503-512, 2007.

13. Singh, D., Coote, M.A., O'Hare, F., et al. Topical prostaglandin analogues do not affect selective laser trabeculoplasty outcomes. Eye (Lond) 23:2194-2199, 2009.

14. Chen, E., Golchin, S., and Blomdahl, S. A comparison between 90 degrees and 180 degrees selective laser trabeculoplasty. J. Glaucoma 13:62-65, 2004.

15. Guex-Crosier, Y., Pittet, N., and Herbort, C.P. Sensitivity of laser flare photometry to monitor inflammation in uveitis of the posterior segment. Ophthalmology 102:613-621, 1995.

16. Herbort, C.P., Guex-Crosier, Y., de Ancos, E., and Pittet, N. Use of laser flare photometry to assess and monitor inflammation in uveitis. Ophthalmology 104:64-71, Discussion 71, 1997.

17. Shah, S.M., Spalton, D.J., and Taylor, J.C. Correlations between laser flare measurements and anterior chamber protein concentrations. Invest. Ophthalmol. Vis. Sci. 33:28782884, 1992.

18. Latina, M.A., Sibayan, S.A., Shin, D.H., Noecker, R.J., and Marcellino, G. Q-switched 532-nm Nd:YAG laser trabeculoplasty (selective laser trabeculoplasty): a multicenter, pilot, clinical study. Ophthalmology 105:2082-2088, Discussion 208, 1998.

19. Arnould, T., Thibaut-Vercruyssen, R., Bouaziz, N., Dieu, M., Remacle, J., and Michiels, C. PGF(2alpha), a prostanoid released by endothelial cells activated by hypoxia, is a chemoattractant candidate for neutrophil recruitment. Am. J. Pathol. 159:345-357, 2001.

20. Hamard, P., Blondin, C., Debbasch, C., Warnet, J.M., Baudouin, C., and Brignole, F. In vitro effects of preserved and unpreserved antiglaucoma drugs on apoptotic marker expression by human trabecular cells. Graefes Arch. Clin. Exp. Ophthalmol. 241:1037-1043, 2003.

21. Miyake, K., and Ibaraki, N. Prostaglandins and cystoid macular edema. Surv. Ophthalmol. 47 Suppl 1:S203-S218, 2002.

22. Negi, A. Latanoprost and cystoid macular edema. J. Cataract Refract. Surg. 29:1055-1056, 2003.

23. Song, J., Lee, P.P., Epstein, D.L., et al. High failure rate associated with 180 degrees selective laser trabeculoplasty. J. Glaucoma 14:400-408, 2005.

24. Melamed, S., Ben Simon, G.J., and Levkovitch-Verbin, H. Selective laser trabeculoplasty as primary treatment for open-angle glaucoma: a prospective, nonrandomized pilot study. Arch. Ophthalmol. 121:957-960, 2003.

25. Aström, S., Stenlund, H., and Lindén, C. Incidence and prevalence of pseudoexfoliations and open-angle glaucoma in northern Sweden: II. Results after 21 years of follow-up. Acta Ophthalmol. Scand. 85:832-837, 2007.

Received: May 9, 2011 Accepted: October 13, 2011

Address correspondence to: Dr. Marcelo Ayala

Department of Glaucoma St. Erik Eye Hospital Karolinska Institutet

Polhemsgatan 50 SE-112 82, Stockholm Sweden

E-mail: marcelo.ayala@sankterik.se 